\title{
The effect of platform switching and crestal placement on marginal bone loss : a systematic review with meta-analysis.
}

IMPLANT THERAPY

OUTCOMES, PERI-IMPLANT BIOLOGY ASPECTS
ITANI Sarah ${ }^{1 *}$, BOILLOT Adrien ${ }^{1 *}$, HAUCHARD Erwan ${ }^{1}$, SAMTMANN Olivier ${ }^{1}$, HENRIOT Edouard ${ }^{1}$, MORET Matthieu ${ }^{1}$, PIRAL Thierry ${ }^{1}$, BENICHOU Ludovic ${ }^{1,2}$.

1. Department of Oral and Maxillofacial Surgery, Saint Joseph Hospital, Paris, France. 2.. Facial Reconstruction Group (FROG), Paris, France. * ITANI Sarah and BOILLOT have equally contributed.

\section{Abstract}

Maintaining marginal bone level is a key factor that contributes to the preservation of peri-implant soft tissues and to the prosthetic success. The aim of this study was to systematically review the combined effect of platform switching with implant placement depth on marginal bone loss

An electronic search was conducted up to March 2017. One-piece implants and immediate post-extractive implants were excluded. The mean differences between groups were calculated using the inverse variance method with a random effects model.

Twenty studies were included in the meta-analysis. More marginal bone loss was observed around platform-matched implants at 12 months after prosthetic loading $(-0.38 \mathrm{~mm}(-0.49 ;-0.27))$. Among platform-switched implants, more marginal bone loss was observed around implants placed subcrestally $(-0.67 \mathrm{~mm}(-1.31 ;-0.03))$.

Further studies are needed to compare marginal bone loss around (1) platform-matched implants placed at different depths and (2) platform-switched implants placed at crestal versus supracrestal levels.

\section{Background and Aim}

Maintaining marginal bone level is a key factor that contributes to the preservation of peri-implant soft tissues and to the prosthetic success. Platform switching has been suggested to preserve marginal bone level. Moreover, subcrestal placement has been proposed to obtain an ideal emergence profile, but it may simultaneously contribute to more marginal bone loss. To date, no study has investigated the combined effect of platform switching with implant placement depth on marginal bone loss. The aim of this study was to systematically review the combined effect of platform switching with implant placement depth on marginal bone loss, from 12 to 18 months after implant placement or prosthetic loading.

\section{Methods and Materials}

An electronic search was conducted up to March 2017. A manual search of implants-related journals was also performed, and authors were contacted to collect unpublished data. This systematic review included clinical trials comparing, for at least 1 year after implant placement, (1) platform-switched versus platform-matched implants placed at same depth, or (2) platformmatched implants placed at different depths, or (3) platformswitched implants placed at different depths. One-piece implants and immediate post-extractive implants were excluded. The mean differences between groups were calculated using the inverse variance method with a random effects model.

\section{Results}

Twenty-nine publications were included in the systematic review, including 1825 platform-switched and 1204 platform-mached implants. More marginal bone loss was observed around platformmatched implants in 28/30 studies. Moreover, marginal bone loss around platform-switched implants decreased when the mismatch increased. (Canullo, 2011. Canullo, 2012)

Most implants were placed at crestal bone level $(n=2036), 661$ were placed subcrestally, and 332 were placed above crestal bone level. When compared with implants placed at crestal bone level, more/less marginal bone loss was observed around implants placed subcrestally/above crestal bone level. Also, for implants placed subcrestally, marginal bone loss increased with implant placement depth. (Koutouzis, 2013)

Twenty studies were included in the meta-analysis. More marginal bone loss was observed around platform-matched implants at 12 months after surgery and prosthetic loading (respectively $-0.20 \mathrm{~mm}$ $(-0.35 ;-0.06)$ and $-0.38 \mathrm{~mm}(-0.49 ;-0.27))$. Among platformswitched implants, more marginal bone loss was observed at 1 year after loading around implants placed subcrestally $(-0.67 \mathrm{~mm}(-1.31$; $-0.03))$.

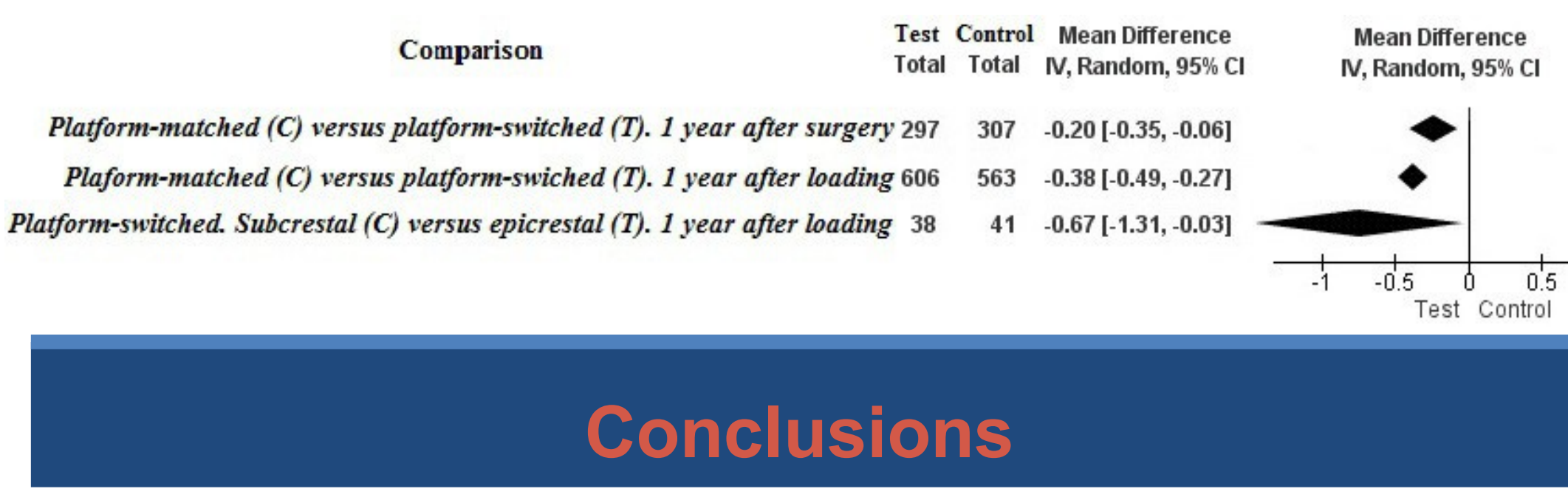

Less marginal bone loss was observed around platform-switched implants which were placed at crestal bone level. Moreover, platform-switched implants that were placed subcrestally exhibited more marginal bone loss than when implants were placed at crestal bone level. Further studies are needed to compare marginal bone loss around (1) platform-matched implants placed at different depths and (2) platform-switched implants placed at crestal versus supracrestal levels.

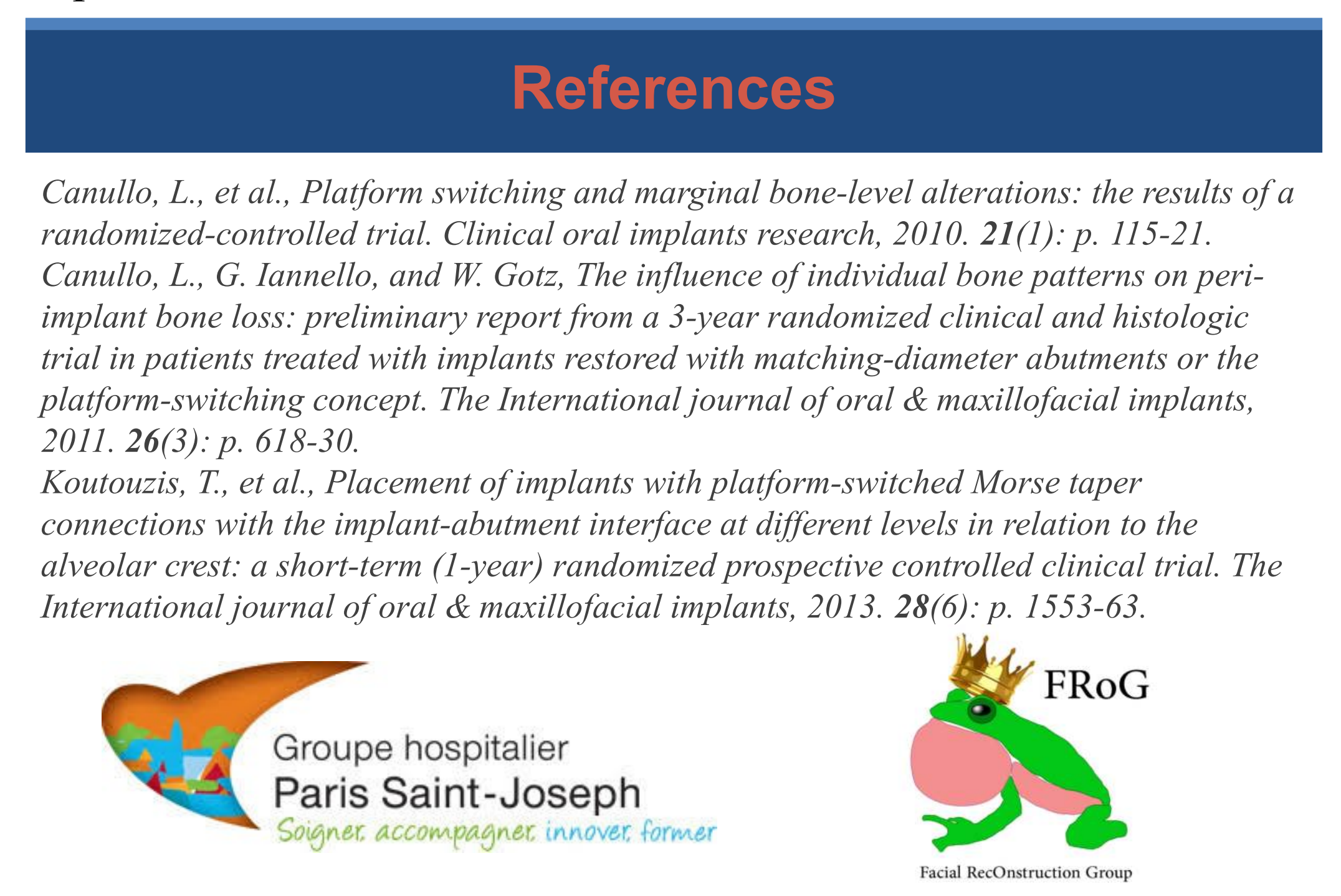

